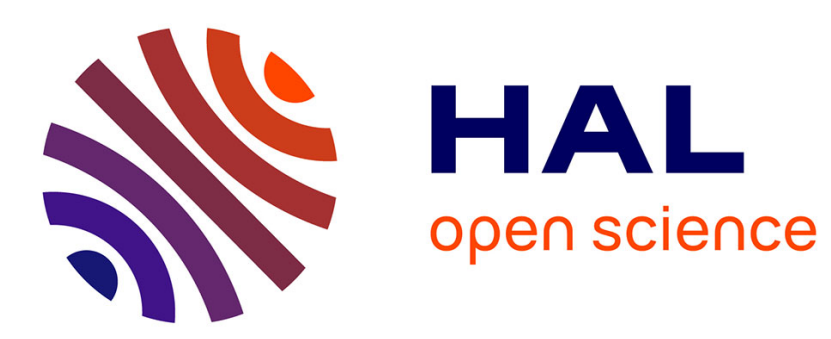

\title{
Optical studies of methane under high pressure
}

P Hebert, A. Polian, P Loubeyre, R Le Toullec

\section{- To cite this version:}

P Hebert, A. Polian, P Loubeyre, R Le Toullec. Optical studies of methane under high pressure. Physical Review B: Condensed Matter (1978-1997), 1987. hal-03059431

\section{HAL Id: hal-03059431 \\ https://hal.science/hal-03059431}

Submitted on 12 Dec 2020

HAL is a multi-disciplinary open access archive for the deposit and dissemination of scientific research documents, whether they are published or not. The documents may come from teaching and research institutions in France or abroad, or from public or private research centers.
L'archive ouverte pluridisciplinaire HAL, est destinée au dépôt et à la diffusion de documents scientifiques de niveau recherche, publiés ou non, émanant des établissements d'enseignement et de recherche français ou étrangers, des laboratoires publics ou privés. 


\title{
Optical studies of methane under high pressure
}

\author{
P. Hebert, ${ }^{*}$ A. Polian, P. Loubeyre, and R. Le Toullec \\ Laboratoire de Physique des Milieux Condensés, Université Pierre et Marie Curie, Tour 13, Etage 4, 4 place Jussieu, \\ F-75252 Paris Cédex 05, France
}

(Received 11 May 1987)

\begin{abstract}
Physical properties of solid methane have been studied at room temperature under high pressure in a diamond-anvil cell. Internal modes have been followed up to $20 \mathrm{GPa}$ by Raman scattering. The refractive index has been measured by a newly developed method up to $12 \mathrm{GPa}$ and the elastic properties determined by Brillouin scattering up to $32 \mathrm{GPa}$. In the plastic phase I, the experimental equation of state and the elastic properties were analyzed with reference to rare-gas solids, i.e., with use of self-consistent harmonic calculations with various pair potentials. The best agreement is obtained with Aziz's Hartree-Fock + dispersion $-C$ krypton-krypton potential. From analogies with rare-gas and $\mathrm{NH}_{3}$ solids, a hcp structure is proposed for phase IV. The IV-VII phase transition is shown to be orientational in nature.
\end{abstract}

\section{INTRODUCTION}

$\mathrm{CH}_{4}$ is the simplest of the saturated hydrocarbon compounds. It is a tetrahedral molecule; in phases without orientational order $\mathrm{CH}_{4}$ may be considered to be spherical and the anisotropy of its intermolecular interactions should be quite small: it could be thus visualized as a "bad rare gas." Moreover, it belongs to the family $\mathrm{CH}_{4}$, $\mathrm{NH}_{3}, \mathrm{H}_{2} \mathrm{O}$, and $\mathrm{HF}$, where the hydrogen interaction replaces the molecular one in the condensed phase as the electronic doublet is substituted for a $\mathrm{H}$ atom; its intensity increases when one goes from methane, where such an interaction is zero, to $\mathrm{HF}$, where it is maximum. Understanding the evolution of such interactions with pressure is of great interest. Finally, methane is an important constituent of our solar system. For these reasons, a number of papers have been devoted to the study of the properties of the phase diagram of methane. ${ }^{1-7} \mathrm{Six}$ solid phases have been discovered at low temperature $(T<80 \mathrm{~K})$ and moderate pressure $(P<1 \mathrm{GPa})$ by various methods. Phase $I$ is fcc with one molecule in the unit cell and belongs to the $F m 3 m\left(O_{h}^{5}\right)$ space group. In this phase, the methane molecules have no orientational order (plastic phase). ${ }^{8}$ Phase II is also fcc with eight molecules in the unit cell and belongs to the $\mathrm{Fm} 3 c\left(O_{h}^{6}\right)$ space group. In this phase, six of the eight molecules are orientationally ordered. ${ }^{8}$ The structure of the other phases is not yet known. Phase III has been calculated to be tetragonal $P_{42} / m b c\left(D_{4 h}^{13}\right)$ and completely ordered, ${ }^{9}$ but this theoretical prediction is in contradiction with recent experimental results. ${ }^{10,11}$ In contrast with the situation at low pressure and low temperature, there are only a few experimental results at room temperature and high pressure. The melting curve has been measured up to $387 \mathrm{~K}$ (Ref. 12) and at $293 \mathrm{~K}$ methane crystallizes around $1.3 \mathrm{GPa}$ into phase $\mathrm{I}$. When the pressure is further increased, a solid-solid phase transition occurs at $5.1 \mathrm{GPa}$ to a still unidentified structure, denoted phase IV. This phase transition has been studied at variable temperature, mainly by NMR, and shows an impor- tant decrease of the hysteresis with increasing temperature. ${ }^{13-15}$ An $x$-ray diffraction study in the diamondanvil cell (DAC) has also been performed up to $5 \mathrm{GPa}$ (Ref. 4), i.e., in phase I. These studies show that phase IV does not belong to the same structure as phase III. ${ }^{16}$

\section{EXPERIMENT}

All the experiments were performed using a BlockPermarini-type DAC. Pure $\mathrm{CH}_{4}$ (purity 99.95\%) was loaded by a cryogenic method. ${ }^{17}$ The pressure was measured using the nonlinear scale for the ruby luminescence, ${ }^{18}$

$$
P=380.8\left[\left[\frac{\lambda}{\lambda_{0}}\right)^{5}-1\right] \mathrm{GPa},
$$

where $\lambda_{0}$ and $\lambda$ are the wavelengths of the $R_{1}$ luminescence line of the ruby chip at zero and high pressure, respectively.

Raman scattering experiments were carried out on a triple monochromator spectrometer, using the 488.0-nm line of an $\mathrm{Ar}^{+}$laser with a power of $120 \mathrm{~mW}$. Resolution was $5 \mathrm{~cm}^{-1}$.

The refractive index has been measured using a new accurate interferometric method developed in our laboratory. ${ }^{19}$

Brillouin scattering experiments were performed using a five-pass Fabry-Pérot interferometer. With our DAC, we are restricted to the backscattering geometry. The exciting light was the $514.5-\mathrm{nm}$ line of a single-moded $\mathrm{Ar}^{+}$laser, with $200 \mathrm{~mW}$ output power.

\section{RESULTS}

\section{A. Raman scattering}

There are nine internal modes of the $\mathrm{CH}_{4}$ molecule: $v_{1}$ is the fully symmetric breathing mode of $A_{1}$ symmetry; $v_{2}$ is doubly degenerate with $E$ symmetry; and $v_{3}$ and $v_{4}$ are triply degenerate with $F_{2}$ symmetry. The 
wave numbers of these modes are at $P=0$ (gas): $\sigma_{1}=2914.2 \mathrm{~cm}^{-1}, \sigma_{2}=1526 \mathrm{~cm}^{-1}, \sigma_{3}=3022 \mathrm{~cm}^{-1}$, and $\sigma_{4}=1306.2 \mathrm{~cm}^{-1}$. In the DAC only three modes $\left(v_{1}, v_{2}\right.$, and $v_{3}$ ) can be studied because $v_{4}$ is hidden behind the very intense first-order Raman mode of the diamond $\left(1331 \mathrm{~cm}^{-1}\right.$ at $P=0$ ). Data were taken up to $20.2 \mathrm{GPa}$ at room temperature. The solid-fluid equilibrium is observed at $P=1.2 \mathrm{GPa}$, in good agreement with previous determinations. ${ }^{12}$ At the fluid-solid equilibrium, methane single crystals do not show any facets and have a spherical shape, like rare-gas crystals. With increasing pressure, the wave number of the $v_{1}$ and $v_{3}$ modes increases with a slope of 11 and $5 \mathrm{~cm}^{-1} / \mathrm{GPa}$, respectively. On the contrary, $v_{2}$ shows only a very small variation with pressure $\left(\approx 0.5 \mathrm{~cm}^{-1} / \mathrm{GPa}\right)$. The I-IV phase transition occurs at $5.1 \pm 0.2 \mathrm{GPa}$. In spite of the small change in refractive index (see below), the transition can easily be observed under a microscope. At room temperature, in contrast with what happens at low temperature, the hysteresis is small. At the transition, there is a sudden change in the slope of the $v_{1}$ and $v_{3}$ modes (Table I). When the pressure is further increased, an accident happens around $12 \mathrm{GPa}$ : in one run the pressure was kept for $2 \mathrm{~h}$ at $12.4 \mathrm{GPa}$, and for $12 \mathrm{~h}$ at $13 \mathrm{GPa}$ in another one. In the first run the wave numbers of $v_{1}$ and $v_{3}$ modes were shifted to higher energies by $4 \mathrm{~cm}^{-1}$ and by $9 \mathrm{~cm}^{-1}$ in the second one. Simultaneously a weak line grew in the low-energy side of the main peak (Fig. 1). At approximately $15 \mathrm{GPa}$, another weak peak appears at still lower energies. It should be noted here that the phenomenon is exactly similar for both $v_{1}$ and $v_{3}$ modes. On the contrary, nothing can be detected for $v_{2}$. No new feature appears when the pressure is further increased up to $20 \mathrm{GPa}$. The main $v_{1}$ and $v_{3}$ peaks have a slightly smaller slope than in phase IV (Table I). When the pressure is decreased, the wave number of the main lines of the $v_{1}$ and $v_{3}$ modes decreases, but stays on the upper line down to $6.6 \mathrm{GPa}$. Down to that pressure, one of the two shoulders is still observable. Figures 2-4 summarize our Raman observations. The behavior of the $v_{1}$ and $v_{3}$ modes is interpreted as a phase transition between the "low"-pressure phase IV and a highpressure phase VII. The hysteresis of the IV-VII phase transition is quite large ( $\approx 6 \mathrm{GPa}$ at room temperature). The nature of this transition will be discussed in Sec. IV.

TABLE I. Pressure dependence (units of $\mathrm{cm}^{-1} / \mathrm{GPa}$ ) of the $v_{1}$ and $v_{3}$ modes as well as their shoulders $v_{1}^{\prime}, v_{1}^{\prime \prime}, v_{3}^{\prime}$, and $v_{3}^{\prime \prime}$ in phase VII, in the three identified room-temperature phases.

\begin{tabular}{lccc}
\hline & & Phase & \\
& I & IV & VII \\
\hline$d \sigma_{1} / d P$ & 10.6 & 7.5 & 7.5 \\
$d \sigma_{1}^{\prime} / d P$ & & & 6.3 \\
$d \sigma_{1}^{\prime \prime} / d P$ & & & 5 \\
$d \sigma_{3} / d P$ & 4.7 & 9.9 & 9 \\
$d \sigma_{3}^{\prime} / d P$ & & & 7.6 \\
$d \sigma_{3}^{\prime \prime} / d P$ & & & 5.4 \\
\hline \hline
\end{tabular}

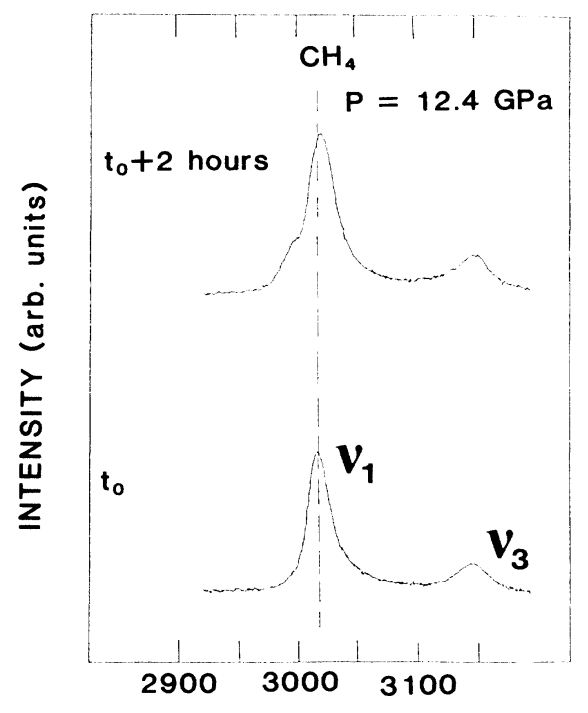

WAVE NUMBER $\left(\mathrm{cm}^{-1}\right)$

FIG. 1. Evolution with time of the $v_{1}$ and $v_{3}$ phonons at $12.4 \mathrm{GPa}$. The lower spectrum shows the peaks just after the pressure was increased and the upper one the same modes two hours later, showing the shift of the main peaks and the appearance of a shoulder in the low-energy side.

\section{B. Refractive index}

The refractive index has been measured up to $12 \mathrm{GPa}$ at room temperature using a new accurate interferometric method. ${ }^{19}$ The relative precision reached with this method is $\Delta n / n \approx 2 \%$. The result of these measurements is shown in Fig. 5. The continuous line is the result of a best fit using a density law for $n$ as explained below and the error bars are shown. It has been

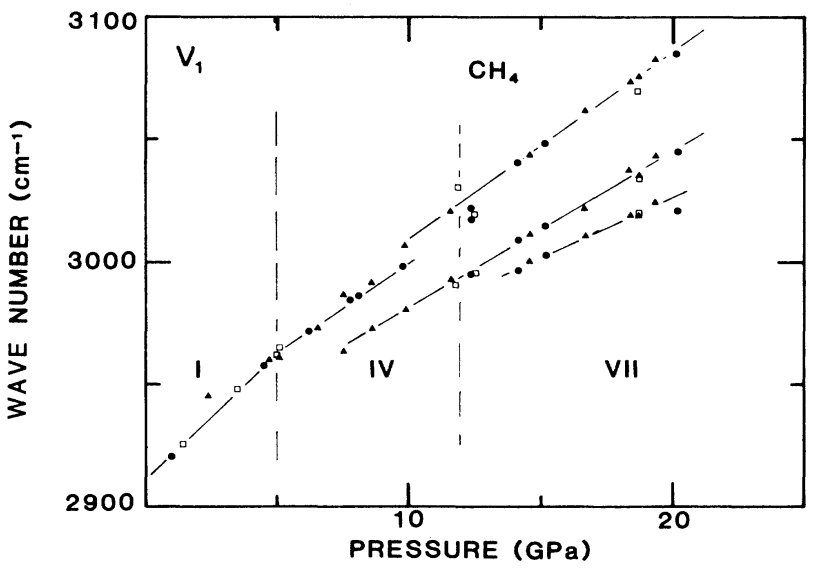

FIG. 2. Summary of the evolution of the $v_{1}$ mode with pressure. The points are obtained at increasing pressure, and the triangles by decreasing it. The open squares are from Ref. 5 . The continuous lines are used to calculate the pressure coefficients given in Table I. The dotted line shows the metastability range of phase VII in the stability range of phase IV. 


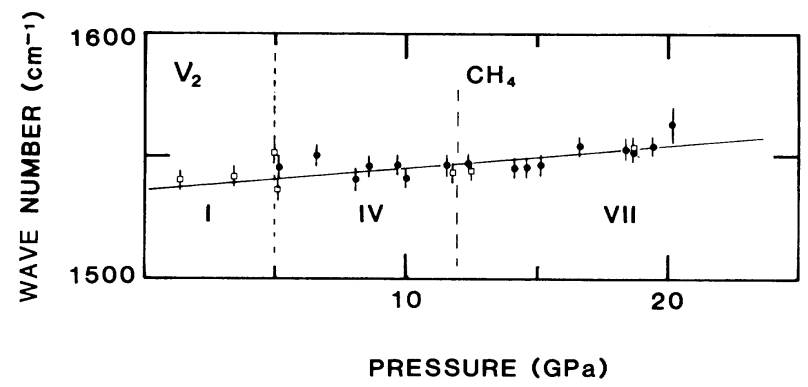

FIG. 3. Same as Fig. 2 for the $v_{2}$ mode. It should be noted that no accident can be detected at the phase transitions.

shown ${ }^{20}$ that the refractive index can be reproduced by a law:

$$
n(P)=n_{0}+\frac{A}{2}\left[1-\left(\frac{\rho}{\rho_{0}}\right)^{2 / 3}\right],
$$

where $n_{0}\left(\rho_{0}\right)$ is the refractive index (the density) at the reference pressure, $\rho$ the density at the pressure $P$, and $A$ is a constant. The $\mathrm{x}$-ray data ${ }^{4}$ have been fitted using a first-order Murnaghan equation of state (EOS):

$$
\frac{\rho}{\rho_{0}}=\left(\frac{B_{0}+B_{0}^{\prime} P}{B_{0}+B_{0}^{\prime} P_{0}}\right)^{1 / B_{0}^{\prime}},
$$

where $B_{0}$ and $B_{0}^{\prime}$ are the bulk modulus and its pressure derivative at zero pressure. The fit was achieved with $B_{0}=4.9 \mathrm{GPa}$ and $B_{0}^{\prime}=4$. At the reference pressure $P_{0}=1.61 \mathrm{GPa}$ the density is $\rho_{0}=0.677 \mathrm{~g} / \mathrm{cm}^{3}$. This EOS has been extrapolated up to $32 \mathrm{GPa}$ in order to interpret our Brillouin data, although there are two phase transitions. Using (3), we have fitted (2) on our results and the best fit (continuous line in Fig. 5) is obtained with $A=-1.433$ and $n_{0}=1.451$. Equation (2) was then extrapolated up to $32 \mathrm{GPa}$ with the same parameters.

\section{Brillouin scattering}

The Brillouin shift has been measured up to $32 \mathrm{GPa}$ in the backscattering geometry. In that geometry, the

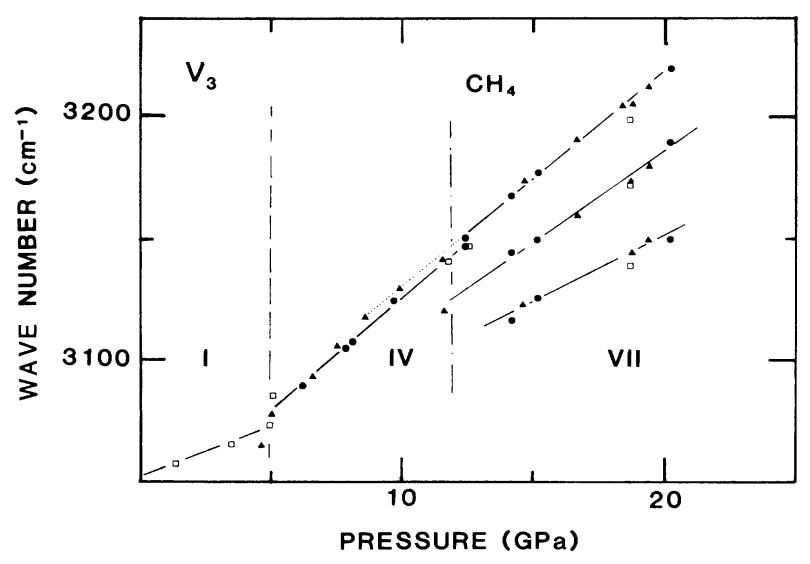

FIG. 4. Same as Fig. 2 for the mode $v_{3}$.

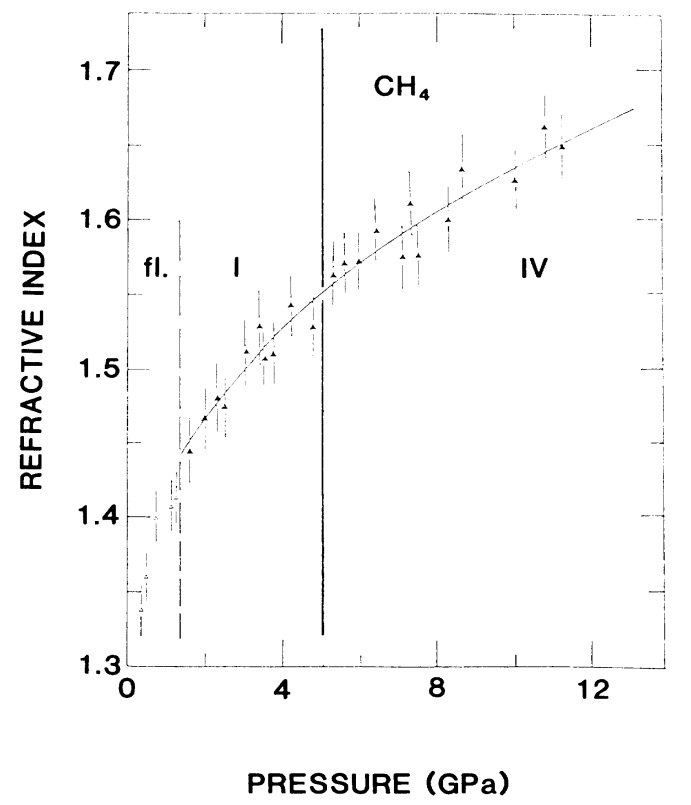

FIG. 5. Refractive index as a function of pressure at $\lambda=514.5 \mathrm{~nm}$ and room temperature. The open triangles are in the fluid and the solid ones are in the solid. The continuous line is the result of the fit using Eq. (2).

wave number shift (in $\mathrm{cm}^{-1}$ ) is related to the sound velocity by

$$
\Delta \sigma=\frac{2 n v}{\lambda c}
$$

where $n$ is the refractive index, $\lambda$ the wavelength of the exciting laser light, $c$ the light velocity in vacuum, and $v$ the sound velocity. The global experimental results are shown in Fig. 6. In phase I there is no dispersion of the experimental points. The I-IV phase transition is accompanied by a $4 \%$ discontinuity (inset in Fig. 6). In phase IV there is also no dispersion of the experimental points. This absence of dispersion in phases I and IV means either that the crystal grows every time with the same orientation relative to the cell axis, or that these

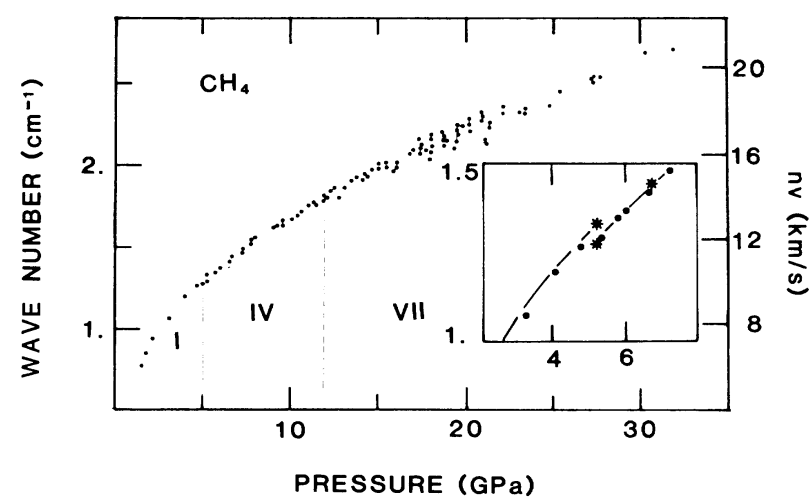

FIG. 6. Brillouin frequency shift as a function of pressure. The inset shows the detail of the I-IV phase transition: the points are at increasing pressure and the stars at decreasing pressure. 
crystals are elastically isotropic. On the contrary, there is an important dispersion above $12 \mathrm{GPa}$. Moreover, between 12 and $25 \mathrm{GPa}$ the measured value depends on the position of the spot in the cell. At higher pressures, this phenomenon disappears. It is thus not related to pressure gradients in the cell, but must be assigned to the progressive appearance of phase VII in the experimental volume. This transition is very sluggish.

From the frequency shift, we deduced the sound velocity using Eq. (4). From $v$, due to the fact that we are not able to determine the crystallographic orientations relative to the scattering geometry, we can only calculate an "effective" elastic modulus which is a linear combination of elastic constants by

$$
C_{\text {eff }}=\rho v^{2} .
$$

\section{Self-consistent harmonic calculations}

In plastic phase $\mathrm{I}$, the $\mathrm{CH}_{4}$ sites have no orientational order and so as a first approximation they can be considered as spherical entities interacting on a fcc lattice. We therefore analyze its properties as was done recently for rare-gas solids at high density. ${ }^{17,21}$ The method used is the self-consistent harmonic theory $(\mathrm{SCH})$ corrected for the cubic anharmonic term near melting; since comprehensive reviews of the $\mathrm{SCH}$ model are available, ${ }^{22,23}$ and since we have used the same numerical implementation as in Ref. 21 , we will here simply recall the basic ideas of the method. In the SCH theory, the force constants used to calculate the dynamical matrix are self-consistently averaged over the positions of the atoms undergoing the thermal motions. It is formulated as a system of coupled equations which are solved iteratively, that takes into account mostly even anharmonic terms. For a better description in the region near melting, we have included the first cubic anharmonic term $\Delta F_{33}$; since it involves a lengthy procedure with a double sum on the Brillouin zone, we calculate it on an Einstein spectrum and add a corrective multiplicative constant which was shown to result in a perfect agreement with Monte Carlo free energies. The sums were carried out over $\frac{1}{48}$ of the total Brillouin zone with a mesh equivalent to 3999 points in the full zone. The frequencies and the free energies converge to within two parts in $10^{4}$ after eight iterations of the self-consistent equations. This model, denoted $\mathrm{SCH}+\mathrm{CE}$, should produce a good description of the EOS and of the elastic constants of dense solid methane. The calculations reported here were performed for three different pair potentials: (i) the potential of Matthews and $\mathrm{Smith}^{24}$ (MS) which was fitted to the second virial coefficient and viscosity measurements; (ii) the potential of Tabisz $^{25}\left(T^{*}\right)$ which is an $a b$ initio pair potential calculated within the electron gas approximation; a Lennard-Jones analytical form is used to represent it; $^{26}$ and (iii) the Hartree-Fock + dispersion $-C(\mathrm{HFD}-\mathrm{C})$ potential of krypton. ${ }^{27}$ Righini et al. ${ }^{28}$ have constructed an atomic site-site potential, the parameters of which were obtained by considering the second virial coefficient of the gas as well as the sublimation energy, molar volume, and $c / a$ ratio of phase
III. They have shown afterwards that the average of the potential taken over the various orientations of the molecules in phase II leads to an isotropic potential which is very similar to the $\mathrm{HFD}-C$ potential of krypton. In Fig. 7 we compare the EOS calculated for these three potentials to the $x$-ray experimental data. Below $5 \mathrm{GPa}$ a fcc structure is assumed for the solid phase. Above that pressure, the calculations were done for both fcc and hcp with practically no differences, which is not surprising since in dense solids, nearest-neighbor interactions are dominant. A Murnaghan EOS fitted on the $\mathrm{x}$ ray results up to $5 \mathrm{GPa}$ is extrapolated with the same parameters. It should not differ significantly from the real one since the refractive index which in a first approximation is proportional to $\rho^{2 / 3}$ does not show any discontinuity at the I-IV phase transition, nor change in regime in its evolution with pressure. In the stability range of phase $I$, the three potentials are somewhat equivalent leading to a good agreement with experiment. At higher pressure, the MS and $T^{*}$ EOS become very different from the "experimental" one and that can be understood in terms of a too hard repulsive part. It seems that the HFD $-C$ potential brings a very good overall agreement up to at least $15 \mathrm{GPa}$. In Fig. 8 we report calculations of the $C_{11}$ elastic constant of the fcc structure for the three potentials; it is obtained from the initial slope of the SCH phonon dispersion curves. Since $C_{11}$ is the minimal value of the longitudinal elastic constant of solid krypton, we compare the calculated $C_{11}$ to the lower bound of the measured elastic constant, which should be greater than or equal to the real $C_{11}$; we can consequently state that the calculated $C_{11}$ are systematically above the real ones, the smallest discrepancy being obtained for the HFD $-C$ potential. In fact our calculations are only justified in phase I (fcc structure). Had we assumed a hcp structure for phase IV, the discrepancy would have been even greater, since at a given pressure

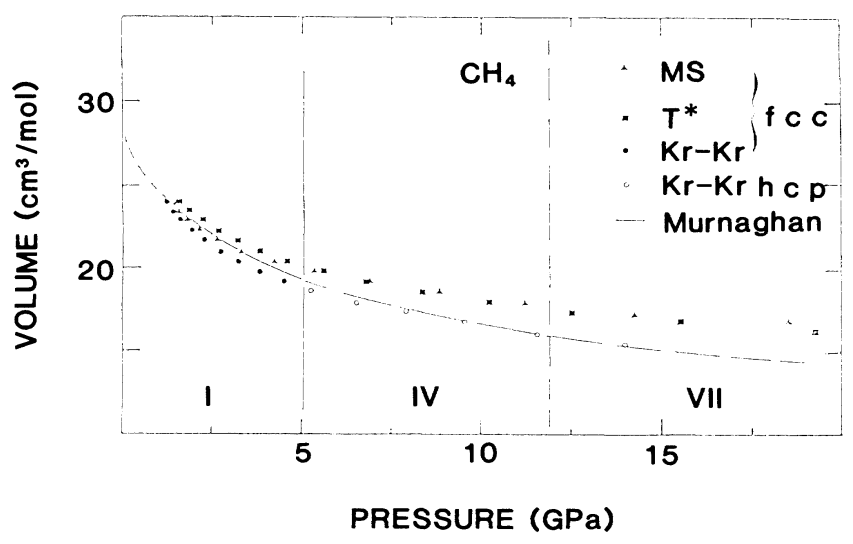

FIG. 7. Comparison between the experimental and calculated EOS. The continuous line is the Murnaghan EOS fitted on the experimental results (Ref. 4) using Eq. (3) and the dashed one its extrapolation in the stability range of phases IV and VII. The calculations were performed in the $\mathrm{SCH}$ phonons formalism using MS (Ref. 24) (triangles), $T^{*}$ (Ref. 25) (squares), and HFD-C krypton-krypton (Ref. 26) (points in the fcc structure and circles in the hcp structure) pair potentials. 


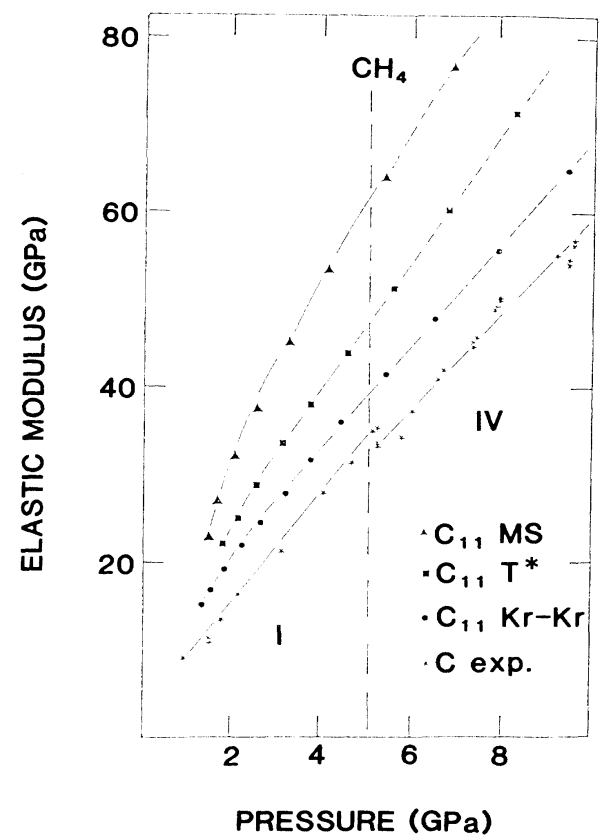

FIG. 8. Comparison of the experimental effective elastic constant with those calculated using MS (triangles), $T^{*}$ (squares), and HFD $-C$ (points) pair potentials.

the minimal longitudinal elastic constant of the two structures belongs to the fcc. From the previous analyses of the EOS and of the elastic constants, it seems that we cannot find a pair potential that reproduces both properties. This is not too surprising since we have neglected the anisotropic effects of the potential. Nevertheless within this approximation, the HFD $-C$ potential is quite good. Solid methane is then very similar to solid krypton. This analogy can be pursued further since, as was recently shown for argon, ${ }^{17}$ at high density the pair potential is no longer sufficient in order to model the interactions of dense rare gases; in that case the departure from the ideal isotropic pair potential interaction is due to three body effects, while in $\mathrm{CH}_{4}$ it is mainly due to the anisotropy of the interactions.

\section{DISCUSSION}

\section{A. I-IV transition}

The structure of phase IV has not yet been determined. From comparisons with more symmetric (krypton) and less symmetric (ammonia) molecules, we propose a structure for phase IV. In the previous section we have shown that $\mathrm{CH}_{4}$ can be considered in a first approximation as a rare-gas-type solid, very similar to krypton, which means that all anisotropy in the interactions is neglected. From another viewpoint, a $\mathrm{NH}_{3} \mathrm{~mol}-$ ecule may be viewed as a $\mathrm{CH}_{4}$ one where one $\mathrm{H}$ atom has been replaced by an electronic doublet. Its solid phase is then composed of pseudo- $\mathrm{CH}_{4}$ molecules with strong anisotropic interactions. Under pressure there is a phase transition at $3.8 \mathrm{GPa}$ from a plastic fcc phase to a hcp plastic with a nonideal $\mathrm{c} / \mathrm{a}$ ratio which, however, evolves with density to the ideal value, as shown by $x$ ray measurements. ${ }^{29}$ These two analogies show that if such a transition takes place in a $\mathrm{CH}_{4}$-type system, increasing or decreasing the anisotropy of the intermolecular interactions leads to high-pressure phases with the same structure, i.e., hcp. But the transition in itself is certainly induced by the anisotropy, since (i) $\mathrm{SCH}$ freeenergy calculations with pure pair potentials do not predict a fcc-hep transition at any pressure, and (ii) in $\mathrm{NH}_{3}$ with larger anisotropy the transition pressure is lower than in $\mathrm{CH}_{4}$.

This assumption is supported by the following experimental results.

(i) There is only one line for each mode in the Raman spectrum in phase IV, as in phase I.

(ii) There is no discontinuity in the wave number of the $v_{1}$ mode at the phase transition, which indicates that the crystal field is very similar in both structures.

(iii) There is a first-order phase transition, proved by the discontinuity in the Brillouin scattering results.

(iv) The volume variation at the transition is small enough to produce in the refractive index a discontinuity much smaller than the error bars.

It should be stressed here that none of the experimental measurements provides a definite characterization of the structure of phase IV. This could be directly probed by $x$-ray diffraction measurements or indirectly by group-theoretical analysis of the present set of data, which would have to be complemented by the measurement of the lattice modes.

In rare-gas solids where such a fcc-hcp transition takes place at high pressure, it is induced by three-body exchange interaction, as recently demonstrated. ${ }^{30}$ It thus seems that the deviation of the interactions from a purely isotropic pair potential description either by anisotropic or many-body effects should stabilize the hcp structure.

\section{B. IV-VII transition}

By measuring at a given pressure the time evolution of the Raman spectrum, we have shown the appearance of phase VII. This appearance is seen by the growing of shoulders on the low-energy side of the $v_{1}$ and $v_{3}$ peaks. The Brillouin scattering study shows that the transition is sluggish and completed only at $25 \mathrm{GPa}$. The intensity of the shoulders increases with pressure. These results suggest that this phase transition could be orientational in nature, leading to a structure with either three distinct sites at $20 \mathrm{GPa}$ or one site and at least three molecules in the unit cell, giving rise to Davydoff multiplets: the free rotations of the molecules in the plastic phase IV are gradually quenched when the pressure is increased. The main peaks of the $v_{1}$ and $v_{3}$ modes would be due to oriented molecules, which would explain the wave-number jump at the transition.

With increasing pressure, the onset of the phase transition is $12 \mathrm{GPa}$, and on decreasing the pressure, phase VII disappears at $P \approx 6 \mathrm{GPa}$. The thermodynamic pressure of transition is then between these two values.

Finally, the actual knowledge of the phase diagram of 
methane is shown in Fig. 9 in semilogarithmic scale, where the points represent the transition pressure measured in the present work.

\section{CONCLUSION}

We have studied methane under pressure up to 32 GPa in a DAC. The wave numbers of the internal modes were measured by Raman scattering, the elastic properties of the solid by Brillouin scattering, and the refractive index by an interferometric method. This set of data confirms the existence of a first-order phase transition I-IV at $5 \mathrm{GPa}$ and a sluggish one, IV-VII, around $10 \mathrm{GPa}$ with a large hysteresis. A self-consistent phonon calculation with the HFD - $C$ krypton pair potential shows that there are strong similarities between phase I of solid methane and solid krypton. Doing then an analogy with krypton on one side and $\mathrm{NH}_{3}$ on the other side in order to test the influence of the anisotropy of the interaction on the nature of phase IV, we get strong confirmation that phase IV should be a plastic one with hcp structure, this structure being stabilized by the anisotropy of the interactions. A direct characterization of phase IV is still certainly needed; this work should stimulate $\mathrm{x}$-ray diffraction measurements. Furthermore, calculations taking into account the anisotropy of the interactions would be most interesting in order to quantitatively probe the conclusions of this work, but they cannot be routinely done and the easiest way seems to be simulations.

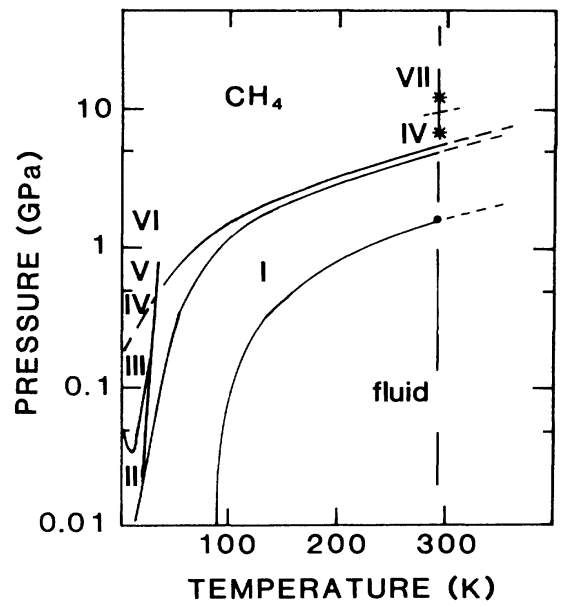

FIG. 9. Proposed phase diagram of methane in semilogarithmic scale. The two stars indicate the onset with increasing pressure and the disappearance by decreasing pressure of phase VII.

\section{ACKNOWLEDGMENTS}

We wish to thank J. M. Besson for a critical reading of the manuscript. This work was supported in part by the Institut National d'Astronomie et de Géphysique under Grant No. 83-070978. The Laboratoire de Physique des Milieux Condensés is "Unité No. 782 associée au Centre National de la Recherche Scientifique."
*Present address: Centre d'Etudes Nucléaires de Saclay, Départment de Physico-Chimie/Section Laser et Optique, Bâtiment 467, F-91191 Gif-sur-Yvette Cédex, France.

${ }^{1}$ M. M. Thiery, D. Fabre, and K. Kobashi, J. Chem. Phys. 83, 6165 (1985).

2J. H. Wieldraaijer, J. A. Schouten, and N. J. Trappeniers, High Temp. High Pressures 15, 87 (1983).

${ }^{3}$ N. J. Trappeniers, in Ices in the Solar System, edited by J. Klinger et al. (Reidel, Dordrecht, 1985), p. 49.

${ }^{4}$ R. M. Hazen, H. K. Mao, L. W. Finger, and P. M. Bell, Appl. Phys. Lett. 37, 288 (1980).

${ }^{5}$ S. K. Sharma, H. K. Mao, and P. M. Bell, Carnegie Institution of Washington Yearbook 79, 351 (1980).

${ }^{6}$ H. J. James and T. A. Keenan, J. Chem. Phys. 31, 12 (1959).

${ }^{7}$ T. Yamamoto, Y. Kataoka, and K. Okada, J. Chem. Phys. 66, 2701 (1977)

${ }^{8}$ W. Press, J. Chem. Phys. 56, 2595 (1972)

${ }^{9}$ K. Maki, Y. Kataoka, and T. Yamamoto, J. Chem. Phys. 66, 655 (1979).

${ }^{10}$ M. Prager, W. Press, and A. Heidemann, J. Chem. Phys. 75, 1442 (1981)

${ }^{11}$ M. Prager, W. Press, A. Heidemann, and C. Vettier, J. Chem. Phys. 77, 2577 (1982).

12J. D. Grace and G. C. Kennedy, J. Phys. Chem. Solids 28, 977 (1967).

${ }^{13}$ A. J. Nijman and N. J. Trappeniers, Physica 95B, 147 (1978).

${ }^{14}$ D. van der Putten, K. O. Prins, and N. J. Trappeniers, Physi- ca 114B, 281 (1982).

${ }^{15}$ D. van der Putten, N. J. Trappeniers, and K. O. Prins, Physica 124B, 193 (1984).

${ }^{16} \mathrm{G}$. Zou, H. K. Mao, and P. M. Bell, Carnegie Institution of Washington Yearbook 81, 391 (1982).

${ }^{17}$ M. Grimsditch, P. Loubeyre, and A. Polian, Phys. Rev. B 33, 7182 (1986).

${ }^{18}$ H. K. Mao, P. M. Bell, J. W. Shaner, and D. J. Steinberg, J. Appl. Phys. 49, 3276 (1978).

${ }^{19}$ M. Grimsditch, R. Le Toullec, A. Polian, and M. Gauthier, J. Appl. Phys. 60, 3479 (1986).

${ }^{20}$ K. Vedam and P. Limsuwan, J. Chem. Phys. 69, 4762 (1978).

${ }^{21}$ P. Loubeyre, D. Levesque, and J. J. Weiss, Phys. Rev. B 33, 318 (1986).

${ }^{22}$ N. R. Werthamer, in Rare Gas Solids, edited by M. Klein and J. A. Venables (Academic, New York, 1976), p. 265.

${ }^{23}$ M. L. Klein and T. R. Koehler, in Rare Gas Solids, Ref. 22, p. 301.

${ }^{24}$ G. P. Matthews and E. B. Smith, Mol. Phys. 32, 1719 (1976).

${ }^{25}$ G. C. Tabisz, Chem. Phys. Lett. 52, 125 (1977).

26J. S. Brown, Phys. Status Solidi B 114, K91 (1982).

${ }^{27}$ R. A. Aziz and H. H. Chen, J. Chem. Phys. 63, 2828 (1975).

${ }^{28}$ R. Righini, K. Maki, and M. L. Klein, Chem. Phys. Lett. 80, 287 (1981).

${ }^{29}$ R. L. Mills (private communication).

${ }^{30} \mathrm{P}$. Loubeyre (unpublished) 